MATHEMATICS OF COMPUTATION

Volume 65, Number 216

October 1996, Pages 1387-1401

\title{
BALANCING DOMAIN DECOMPOSITION FOR PROBLEMS WITH LARGE JUMPS IN COEFFICIENTS
}

\author{
JAN MANDEL AND MARIAN BREZINA
}

\begin{abstract}
The Balancing Domain Decomposition algorithm uses in each iteration solution of local problems on the subdomains coupled with a coarse problem that is used to propagate the error globally and to guarantee that the possibly singular local problems are consistent. The abstract theory introduced recently by the first-named author is used to develop condition number bounds for conforming linear elements in two and three dimensions. The bounds are independent of arbitrary coefficient jumps between subdomains and of the number of subdomains, and grow only as the squared logarithm of the mesh size $h$. Computational experiments for two- and three-dimensional problems confirm the theory.
\end{abstract}

\section{INTRODUCTION}

Domain decomposition methods for solving elliptic boundary value problems have received much attention in the last few years. The main reason for the popularity of these methods is undoubtedly the need to take advantage of parallel computers, but many domain decomposition methods are efficient solvers in a classical uniprocessor environment as well. This paper is concerned with a domain decomposition preconditioner for linear, conforming finite elements for the elliptic equation $-\nabla \cdot(\sigma \nabla u)=f$ with the coefficients $\sigma$ changing between the subdomains by orders of magnitude.

The main component of the domain decomposition algorithms of the type studied here is an approximate solver based on the solution of local independent subproblems on subdomains and a global coarse problem with one or a few unknowns for each subdomain to effect a global exchange of information between the subdomain solution. The composed approximate solver is then used as a preconditioner in the conjugate gradients method. It is well known that the absence of a coarse problem results in deterioration of convergence of the iterations with increasing number of subdomains $[11,14]$.

The Balancing Domain Decomposition (BDD) was introduced by Mandel [20] by adding a coarse problem to an earlier method of De Roeck and Le Tallec [11], known as the Neumann-Neumann method, based in turn on earlier work for the case of two subdomains [2] and on a closely related method of Glowinski and Wheeler for mixed

Received by the editor March 18, 1993 and, in revised form, December 2, 1993 and September 21, 1994.

1991 Mathematics Subject Classification. Primary 65N55, 65F10.

Key words and phrases. Domain decomposition, second-order elliptic boundary value problems, two-level iterative methods, discontinuous coefficients.

Submitted March 1993; revised September 1994. 
problems [17]. The development of BDD was motivated by very good performance of the Neumann-Neumann preconditioner for real-world problems with strongly discontinuous coefficients for a small number of subdomains [11]. Algorithms similar to BDD but different in important aspects and convergence results also independent of coefficient jumps between subdomains were recently obtained by Dryja and Widlund [16], and by Sarkis [23]. For application of the BDD method to mixed finite elements and computational results on a parallel computer, see Cowsar, Mandel, and Wheeler [9]. For extensions of BDD to plate bending and performance results on a shared-memory parallel machine, see Le Tallec, Mandel, and Vidrascu [19].

In this paper, we extend the abstract theory from [20] by an elementary argument showing that the convergence of the BDD method is bounded independently on coefficient jumps of arbitrary size between subdomains. We obtain new bounds on convergence from the abstract theory by unifying the fundamental inequalities of the Domain Decomposition theory by Dryja and Widlund [12, 13, 26, 27] and Bramble, Pasciak, and Schatz $[3,5]$, and complementing them with some new results in the $2 \mathrm{D}$ case. In the $3 \mathrm{D}$, we only need to use the inequalities from [5]. We show that the condition number after preconditioning is bounded by $C\left(1+\log ^{2}(H / h)\right)$, where $H$ is the characteristic subdomain size and $h$ is the characteristic element size, both in $2 \mathrm{D}$ and $3 \mathrm{D}$. In the $3 \mathrm{D}$ case, such a bound was already given in [20] based on a different estimate from [11]. The theory is confirmed by computational experiments. Further numerical results, available in the technical report [21], demonstrate that the method behaves very well even in the case of general discontinuities of the coefficients and irregular subdomain shapes in 2D and in many cases in 3D as well.

The paper is organized as follows: $\S 2$ introduces the BDD algorithm. Abstract bounds on the condition number are given in $\S 3$, relying only on algebraic arguments. The assumptions of these bounds are verified for finite element discretizations in $\S 4$. Finally, $\S 5$ contains numerical results.

\section{Formulation of the Problem AND ALGORithms}

We will recall the notation and formulation of the algorithm, following [20]. Consider a system of linear algebraic equations

$$
A x=f,
$$

with the $m \times m$, symmetric positive definite matrix $A$ arising from a finite element discretization of a linear, elliptic, self-adjoint boundary value problem on a domain $\Omega$. We assume the domain $\Omega$ to be split into nonoverlapping subdomains $\Omega_{1}, \ldots, \Omega_{k}$, each of which is the union of some of the elements. Let $A_{i}$ be the local stiffness matrix corresponding to subdomain $\Omega_{i}, x_{i}$ be the vector of degrees of freedom corresponding to all elements in $\Omega_{i}$, and let $N_{i}$ denote the matrix with entries 0 or 1 mapping the degrees of freedom $x_{i}$ into global degrees of freedom, i.e., $x_{i}=N_{i}^{T} x$. Then the stiffness matrix $A$ is obtained by the standard subassembly process,

$$
A=\sum_{i=1}^{k} N_{i} A_{i} N_{i}^{T} .
$$

Each $x_{i}$ is split into degrees of freedom $\bar{x}_{i}$ that correspond to $\partial \Omega_{i}$, called interface degrees of freedom, and the remaining interior degrees of freedom $\dot{x}_{i}$. The degrees of freedom on $\partial \Omega \cap \partial \Omega_{i}$ are assigned to the interiors. The subdomain stiffness matrices 
and the 0-1 matrices $N_{i}$ are then split accordingly:

$$
x_{i}=\left(\begin{array}{c}
\bar{x}_{i} \\
\dot{x}_{i}
\end{array}\right), \quad A_{i}=\left(\begin{array}{cc}
\bar{A}_{i} & B_{i} \\
B_{i}^{T} & \dot{A}_{i}
\end{array}\right), \quad N_{i}=\left(\bar{N}_{i}, \dot{N}_{i}\right) .
$$

Assume the subdomain matrices $A_{i}$ to be symmetric and positive semidefinite and the submatrices $\dot{A}_{i}$ nonsingular. Without loss of generality, let the interface degrees of freedom be numbered first and the interior degrees of freedom second in the global numbering. Let

$$
\Gamma=\bigcup_{i=1}^{k} \partial \Omega_{i},
$$

$V_{i}$ be the space of the interface degrees of freedom for the subdomain $\Omega_{i}$ and $V$ denote the space of all degrees of freedom on $\Gamma$, in a global numbering. After elimination of the interior degrees of freedom, the problem (1) reduces to a problem posed on the interface space $V$,

$$
S u=g,
$$

where $S$ is the Schur complement

$$
S=\sum_{i=1}^{k} \bar{N}_{i} S_{i} \bar{N}_{i}^{T}, \quad S_{i}=\bar{A}_{i}-B_{i} \dot{A}_{i}^{-1} B_{i}^{T} .
$$

The local Schur complements $S_{i}$ are symmetric positive semidefinite and $S$ is positive definite. Interpreting matrices as mappings, we have

$$
S: V \rightarrow V, \quad S_{i}: V_{i} \rightarrow V_{i}, \quad \bar{N}_{i}: V_{i} \rightarrow V .
$$

Throughout this paper, we denote $\langle u, v\rangle=u^{T} v$ and, for symmetric positive semidefinite $B,\langle u, v\rangle_{B}=\langle B u, v\rangle$ and $\|u\|_{B}=\left(\langle u, u\rangle_{B}\right)^{1 / 2}$. The notation $u \perp v$ means $\langle u, v\rangle=0$.

Much of the benefit of domain decomposition is obtained already by solving the reduced problem (2) by conjugate gradients with simple preconditioners such as an approximation to the diagonal of $S$, cf. $[6,7,18]$. Evaluation of the action of $S_{i}$ can be implemented by solving a Dirichlet problem on $\Omega_{i}$. The BDD method is based on the Neumann-Neumann preconditioner [11, 10], which requires the solution of a Neumann problem on every subdomain $\Omega_{i}$ (named so in contrast to the Neumann-Dirichlet preconditioner, which requires solving Neumann problems on some subdomains and uses the original Dirichlet problem on others).

An important design choice for the Neumann-Neumann preconditioner is the selection of weight matrices $D_{i}$ that form a decomposition of unity on the interface space $V$,

$$
\sum_{i=1}^{k} \bar{N}_{i} D_{i} \bar{N}_{i}^{T}=I
$$

A straightforward choice for $D_{i}$ is a diagonal matrix with the diagonal elements being the reciprocal of the number of subdomains the degree of freedom is associated with. A better choice, which also guarantees a convergence bound independent of coefficient jumps between subdomains, is given in Theorem 3.3 below. For other possibilities, see [11] and $\S 5$ below.

The following algorithm defines a linear operator $M_{\mathrm{N}-\mathrm{N}}^{-1}$ for use as a preconditioner. 
Algorithm 2.1 (Neumann-Neumann preconditioner, [11]). Given $r \in V$, compute $z=M_{\mathrm{N}-\mathrm{N}}^{-1} r$ as follows. Distribute $r$ to subdomains,

$$
r_{i}=D_{i}^{T} \bar{N}_{i}^{T} r
$$

solve the local problems

$$
S_{i} u_{i}=r_{i}
$$

on the subdomains, and average the results by

$$
z=\sum_{i=1}^{k} \bar{N}_{i} D_{i} u_{i}
$$

Since the matrices $S_{i}$ are typically singular, De Roeck and Le Tallec [11] used a pseudoinverse obtained by replacing zero pivots in the Gaussian decomposition by positive values.

The BDD method adds a coarse problem as follows. Let $n_{i}=\operatorname{dim} V_{i}, 0 \leq m_{i} \leq$ $n_{i}$, and $Z_{i}$ be $n_{i} \times m_{i}$ matrices of full column rank such that

$$
\text { Ker } S_{i} \subset \text { Range } Z_{i}, \quad i=1, \ldots, k,
$$

and let $W \subset V$ be defined by

$$
W=\left\{v \in V \mid v=\sum_{i=1}^{k} \bar{N}_{i} D_{i} u_{i}, u_{i} \in \text { Range } Z_{i}\right\} .
$$

The space $W$ will play the role of a coarse space just as in variational multigrid methods [22]. We say that $s \in V$ is balanced if

$$
Z_{i}^{T} D_{i}^{T} \bar{N}_{i}^{T} s=0, \quad i=1, \ldots, k .
$$

The process of replacing $r$ by a balanced $s=r-S w, w \in W$, will be called balancing. We are now ready to define the action $r \mapsto z=M^{-1} u$ of the BDD preconditioner.

Algorithm 2.2 (BDD preconditioner, [20]). Given $r \in V$, compute $M^{-1} r$ as follows. Balance the original residual by solving the auxiliary problem for unknown vectors $\lambda_{i} \in \mathbb{R}^{m_{i}}$,

$$
Z_{i}^{T} D_{i}^{T} \bar{N}_{i}^{T}\left(r-S \sum_{j=1}^{k} \bar{N}_{j} D_{j} Z_{j} \lambda_{j}\right)=0, \quad i=1, \ldots, k,
$$

and set

$$
s=r-S \sum_{j=1}^{k} \bar{N}_{j} D_{j} Z_{j} \lambda_{j}, \quad s_{i}=D_{i}^{T} \bar{N}_{i}^{T} s, \quad i=1, \ldots, k .
$$

Find any solution $u_{i}$ for each of the local problems

$$
S_{i} u_{i}=s_{i}, \quad i=1, \ldots, k,
$$

balance the residual by solving the auxiliary problem for $\mu_{i} \in \mathbb{R}^{m_{i}}$,

$$
Z_{i}^{T} D_{i}^{T} \bar{N}_{i}^{T}\left(r-S \sum_{j=1}^{k} \bar{N}_{j} D_{j}\left(u_{j}+Z_{j} \mu_{j}\right)\right)=0, \quad i=1, \ldots, k
$$


and average the result on the interfaces according to

$$
z=\sum_{i=1}^{k} \bar{N}_{i} D_{i}\left(u_{i}+Z_{i} \mu_{i}\right)
$$

If some $m_{i}=0$, then $Z_{i}$ as well as the block unknowns $\mu_{i}$ and $\lambda_{i}$ are void and the $i$ th block equation is taken out of (9) and (12). The presence of the coarse problem now guarantees that the possibly singular local problems (11) are consistent, and the result of the algorithm does not depend on the choice of the solutions of (11), see $[20]$.

In practice, the residual of the initial approximation should be balanced first as in (12); then the first balancing step (9) in every iteration can be omitted since the residual $r$ received from the conjugate gradients algorithm is already balanced.

\section{Algebraic theory}

In this section, we give bounds on the condition number, relying on algebraic arguments only. These results apply to arbitrary linear systems of the form described in the preceding section. Their assumptions will be verified in the following section for systems obtained from a particular variant of the Finite Element Method.

The following theorem was proved in [20, Theorem 3.2] in the case when Range $Z_{i}$ $=\operatorname{Ker} S_{i}$, but the same proof applies here.

Theorem 3.1. Algorithm 2.2 returns $z=M^{-1} r$, where $M$ is symmetric positive definite and cond $(M, S)=\lambda_{\max }\left(M^{-1} S\right) / \lambda_{\min }\left(M^{-1} S\right) \leq C$, where

$C=\sup \left\{\frac{\sum_{j=1}^{k}\left\|\bar{N}_{j}^{T} \sum_{i=1}^{k} \bar{N}_{i} D_{i} u_{i}\right\|_{S_{j}}^{2}}{\sum_{i=1}^{k}\left\|u_{i}\right\|_{S_{i}}^{2}} \mid u_{i} \in V_{i}, u_{i} \perp\right.$ Ker $S_{i}, S_{i} u_{i} \perp$ Range $\left.Z_{i}\right\}$.

To motivate the bound given in Theorem 3.1, we need the concepts of glob and glob projection, defined as follows.

Definition 3.2. Any vertex, edge, and, in the 3D case, face, of $\Gamma$ will be called a glob. A glob is understood to be relatively open; for example, an edge does not contain its endpoints. We will also identify a glob with the set of the degrees of freedom associated with it. The set of all globs will be denoted by $\mathcal{G}$. For a glob $G \in \mathcal{G}$, define the glob projection as follows: for a vector $u \in V, E_{G} u \in V$ is the vector that has the same values as $u$ for all degrees of freedom in $G$, and all other degrees of freedom of $E_{G} u$ are zero. The glob projection in terms of the local degrees of freedom is $E_{G}^{j i}=\bar{N}_{j}^{T} E_{G} \bar{N}_{i}: V_{i} \rightarrow V_{j}$.

Note that any two distinct globs from $\mathcal{G}$ are disjoint, and $\Gamma=\bigcup_{i=1}^{k} \partial \Omega_{i}=$ $\bigcup_{G \in \mathcal{G}} G$. The mappings $E_{G}, E_{G}^{i j}$ correspond to zero-one matrices and satisfy

$$
\sum_{G \in \mathcal{G}} E_{G}=I, \quad \bar{N}_{j}^{T} \bar{N}_{i}=\sum_{G \in \mathcal{G}} E_{G}^{j i}, \quad E_{G}^{j i}=E_{G}^{j i} E_{G}^{i i},
$$

and

$$
G \subset \partial \Omega_{i} \cap \partial \Omega_{j} \Longleftrightarrow E_{G}^{j i} \neq 0, \quad G \subset \partial \Omega_{i} \Longleftrightarrow E_{G}^{i i} \neq 0 .
$$

We are now ready for an abstract bound in the case when the matrices $S_{i}$ are scaled by arbitrary positive numbers $\alpha_{i}$, which corresponds to coefficient discontinuities of arbitrary size between the subdomains. The theorem is formulated and proved in terms of properties of matrices only. 
Theorem 3.3. Let $\alpha_{i}>0, i=1, \ldots, k, t \geq 1 / 2$, and $E_{G}^{j i}, \bar{N}_{i}, S_{i}$, and $Z_{i}$ satisfy (3), (14), and (7). Define $D_{i}$ as the diagonal matrices

$$
D_{i}=\sum_{G: E_{G}^{i i} \neq 0} d(i, G) E_{G}^{i i}, \quad d(i, G)=\frac{\alpha_{i}^{t}}{\sum_{j: E_{G}^{j i} \neq 0} \alpha_{j}^{t}}
$$

and assume that there exists a number $R$ so that for all $i, j=1, \ldots, k$ and all $G$,

$$
\frac{1}{\alpha_{j}}\left\|E_{G}^{j i} u_{i}\right\|_{S_{j}}^{2} \leq \frac{1}{\alpha_{i}} R\left\|u_{i}\right\|_{S_{i}}^{2}
$$

for all $u_{i}$ such that $u_{i} \perp \operatorname{Ker} S_{i}, S_{i} u_{i} \perp$ Range $Z_{i}$. Then the weight matrices $D_{i}$ form a decomposition of unity (5), and the preconditioner defined by Algorithm 2.2 satisfies

$$
\text { cond }(M, S) \leq K^{2} L^{2} R,
$$

where $K=\max _{i}\left|\left\{j \mid \bar{N}_{j}^{T} \bar{N}_{i} \neq 0\right\}\right|$, and $L=\max _{i, j}\left|\left\{G \mid E_{G}^{j i} \neq 0\right\}\right|$.

Proof. The property (5) follows from the definition (16) and from (14),

$$
\sum_{i=1}^{k} \bar{N}_{i}^{T} D_{i} \bar{N}_{i}=\sum_{i=1}^{k} \sum_{G: E_{G}^{i i} \neq 0} d(i, G) E_{G}=\sum_{G \in \mathcal{G}} E_{G}=I
$$

Let $j$ be fixed. Since there are at most $K$ nonzero terms in the $\operatorname{sum} \sum_{i=1}^{k} \bar{N}_{j}^{T} \bar{N}_{i} D_{i} u_{i}$, it follows by the triangle inequality and the Cauchy inequality that

$$
\left\|\sum_{i=1}^{k} \bar{N}_{j}^{T} \bar{N}_{i} D_{i} u_{i}\right\|_{S_{j}}^{2} \leq\left(\sum_{i=1}^{k}\left\|\bar{N}_{j}^{T} \bar{N}_{i} D_{i} u_{i}\right\|_{S_{j}}\right)^{2} \leq K \sum_{i=1}^{k}\left\|\bar{N}_{j}^{T} \bar{N}_{i} D_{i} u_{i}\right\|_{S_{j}}^{2},
$$

and

$$
\sum_{j=1}^{k}\left\|\sum_{i=1}^{k} \bar{N}_{j}^{T} \bar{N}_{i} D_{i} u_{i}\right\|_{S_{j}}^{2} \leq K^{2} \sum_{i=1}^{k} \max _{j}\left\|\bar{N}_{j}^{T} \bar{N}_{i} D_{i} u_{i}\right\|_{S_{j}}^{2} .
$$

If $E_{G}^{j i} \neq 0$, the coefficient $d(i, G)$ from (16) satisfies $d(i, G) \leq \alpha_{i}^{t} /\left(\alpha_{i}^{t}+\alpha_{j}^{t}\right)$, and it follows from (14) and from (17) that

$$
\begin{aligned}
\left\|\bar{N}_{j}^{T} \bar{N}_{i} D_{i} u_{i}\right\|_{S_{j}} & \leq \sum_{G: E_{G}^{j i} \neq 0} \frac{\alpha_{i}^{t}}{\alpha_{i}^{t}+\alpha_{j}^{t}}\left\|E_{G}^{j i} u_{i}\right\|_{S_{j}} \leq \sum_{G: E_{G}^{j i} \neq 0} \frac{\alpha_{i}^{t-1 / 2} \alpha_{j}^{1 / 2}}{\alpha_{i}^{t}+\alpha_{j}^{t}} R^{1 / 2}\left\|u_{i}\right\|_{S_{i}} \\
& \leq L R^{1 / 2} \sup _{\rho>0} \frac{\rho^{1 / 2}}{1+\rho^{t}}\left\|u_{i}\right\|_{S_{i}} \leq L R^{1 / 2}\left\|u_{i}\right\|_{S_{i}} .
\end{aligned}
$$

Now by (19),

$$
\sum_{j=1}^{k}\left\|\sum_{i=1}^{k} \bar{N}_{j}^{T} \bar{N}_{i} D_{i} u_{i}\right\|_{S_{j}}^{2} \leq K^{2} L^{2} R\left\|u_{i}\right\|_{S_{i}}^{2},
$$

which concludes the proof, owing to Theorem 3.1.

Note that the constant $K$ is the maximal number of adjacent subdomains $\Omega_{j}$ to any subdomain $\Omega_{i}$ plus one, and $L$ is the maximal number of globs in any $\partial \Omega_{i} \cap \partial \Omega_{j}$. If $t>1 / 2$, the estimate (18) can be slightly improved; in particular, if $t=1$, analogously to the method of De Roeck and Le Tallec [11], one has cond $(M, S) \leq K^{2} L^{2} R / 2$. 
The related method of Dryja and Widlund [16] uses the coarse space $W$ with $t=1 / 2$ in (16), and the matrices $S_{i}$ in (11) replaced by $S_{i}+c_{i} M_{i}, M_{i}$ positive definite, to avoid solving singular problems. Sarkis [23] obtained an estimate for a similar method for nonconforming elements with any $t \geq 1 / 2$.

\section{ThEORY FOR A FINITE ELEMENT DiscRETIZATION}

Let $\Omega$ be a bounded domain in $\mathbb{R}^{d}(d=2$ or $d=3)$ with a piecewise smooth boundary $\partial \Omega$, and $\partial \Omega=\Gamma_{1} \cup \Gamma_{2}$ with $\Gamma_{1}, \Gamma_{2}$ disjoint, $\left|\Gamma_{1}\right|>0$. Consider the model problem

$$
L u=f \text { in } \Omega, \quad u=g \text { on } \Gamma_{1}, \quad \frac{\partial u}{\partial n}=0 \text { on } \Gamma_{2},
$$

where

$$
L v=-\sum_{r, s=1}^{d} \frac{\partial}{\partial x_{r}}\left(\alpha(x) \beta_{r s}(x) \frac{\partial v(x)}{\partial x_{s}}\right)
$$

with the coefficient matrix $\left(\beta_{r s}\right)$ uniformly positive definite, bounded and piecewise smooth on $\Omega$, and $\alpha(x)$ a positive constant in each subdomain $\Omega_{i}$, i.e.,

$$
\alpha(x)=\alpha_{i}>0 \quad \text { for } x \in \Omega_{i} .
$$

Let $\hat{\Omega}$ denote a reference domain of diameter $O(1)$ (e.g., square or cube in $2 \mathrm{D}$ or $3 \mathrm{D}$, respectively) and assume that the subdomains $\Omega_{i}$ are of diameter $O(H)$ and shape regular, i.e.,

$$
\Omega_{i}=F_{i}(\hat{\Omega}), \quad\left\|\partial F_{i}\right\| \leq C H, \quad\left\|\partial F_{i}^{-1}\right\| \leq C H^{-1},
$$

with $\partial F_{i}$ the Jacobian and $\|\cdot\|$ the Euclidean $\mathbb{R}^{d}$ matrix norm.

Let $V_{h}(\Omega)$ be a conforming linear finite element space on a triangulation of $\Omega$ such that each subdomain $\Omega_{i}$ is the union of some of the elements, and the usual shape regularity and inverse assumption hold [8]. All functions $v \in V_{h}(\Omega)$ satisfy homogeneous boundary condition $u=0$ on $\Gamma_{1}$.

Let $V_{h}\left(\Omega_{i}\right)$ be the space of the restrictions of functions in $V_{h}(\Omega)$ to $\Omega_{i}$. In all the estimates below, $C$ and $c$ denote generic positive constants independent of the shape or size of $\Omega$ and $\Omega_{i}$. Note that these constants may depend on the constant in (22) or on the regularity of the triangulation, but they are independent of $h$ and $H$.

Following [4], [12] or [25], we define the scaled Sobolev norms

$$
\|u\|_{1, \Omega_{i}}^{2}=|u|_{1, \Omega_{i}}^{2}+\frac{1}{H^{2}}|u|_{0, \Omega_{i}}^{2}, \quad\|u\|_{1 / 2, \partial \Omega_{i}}^{2}=|u|_{1 / 2, \partial \Omega_{i}}^{2}+\frac{1}{H}|u|_{0, \partial \Omega_{i}}^{2},
$$

where

$$
|u|_{1, \Omega_{i}}^{2}=\int_{\Omega_{i}}|\nabla u(x)|^{2} d x, \quad|u|_{1 / 2, \partial \Omega_{i}}^{2}=\int_{\partial \Omega_{i}} \int_{\partial \Omega_{i}} \frac{|u(t)-u(s)|^{2}}{|t-s|^{d}} d t d s .
$$

The advantage of this definition is that it allows us to restrict all of our considerations to the reference domain $\hat{\Omega}$ and use the mappings $F_{i}$ to obtain the results for each $\Omega_{i}$ from the obvious norm equivalence

$$
\begin{gathered}
c\|u\|_{1, \Omega_{i}}^{2} \leq\left\|u \circ F_{i}\right\|_{1, \hat{\Omega}}^{2} H^{d-2} \leq C\|u\|_{1, \Omega_{i}}^{2}, \\
c\|u\|_{1 / 2, \partial \Omega_{i}}^{2} \leq\left\|u \circ F_{i}\right\|_{1 / 2, \partial \hat{\Omega}}^{2} H^{d-2} \leq C\|u\|_{1 / 2, \partial \Omega_{i}}^{2}
\end{gathered}
$$


Assume that for each $\Omega_{i}, \Gamma_{1} \cap \partial \Omega_{i}$ is either empty or a part of $\partial \Omega_{i}$ of size bounded below by a fixed proportion of the size of $\partial \Omega_{i}$ so that the Poincaré inequality holds uniformly for all $\Omega_{i}$ and with the constant $C$ independent of $h$ and $H$,

$$
|u|_{0, \Omega_{i}}^{2} \leq C H|u|_{1, \Omega_{i}}^{2}, \quad|u|_{0, \partial \Omega_{i}}^{2} \leq C H^{1 / 2}|u|_{1 / 2, \partial \Omega_{i}}^{2}
$$

for all $u \in V_{h}\left(\Omega_{i}\right)$ if $\Gamma_{1} \cap \partial \Omega_{i} \neq \varnothing$ and for all $u \in V_{h}\left(\Omega_{i}\right), \int_{\partial \Omega_{i}} u d s=0$ if $\Gamma_{1} \cap \partial \Omega_{i}=\varnothing$.

To apply Theorem 3.1, we first need to replace the $S_{i}$ norm by the scaled $H^{1 / 2}$ norm. This is a standard result $[3,13,26]$, which we state here for reference in a form suitable for our purposes. The scaling by $\alpha_{i}$ is obvious.

Lemma 4.1. There exist constants $c>0, C$ independent of $H$ or $h$ so that

$$
c|u|_{1 / 2, \partial \Omega_{i}}^{2} \leq \frac{1}{\alpha_{i}}\|u\|_{S_{i}}^{2} \leq C|u|_{1 / 2, \partial \Omega_{i}}^{2}, \quad \forall u \in V_{h}\left(\partial \Omega_{i}\right) .
$$

To derive the fundamental inequality (17) assumed in Theorem 3.3, we identify (by abuse of notation) $V$ with $V_{h}(\Gamma)$ and $V_{i}$ with $V_{h}\left(\partial \Omega_{i}\right)$. Then the glob projections are $E_{G}: V_{h}(\Gamma) \rightarrow V_{h}(\Gamma)$, and (17) becomes a bound on the increase of the $H^{1 / 2}$ norm when a function in $V_{h}\left(\partial \Omega_{i}\right)$ is changed by setting its values to zero on all nodes of $\partial \Omega_{i} \backslash G$.

We first consider the two-dimensional case, $\Omega \subset \mathbb{R}^{2}$. Since $\partial \Omega_{i}$ is one-dimensional, we may use the properties of the space $V_{h}(0, H)$ of piecewise linear functions on a uniform mesh with step $h$ on the interval $[0, H]$. The following form of Discrete Sobolev Inequality was proved by Dryja [12].

Lemma 4.2. There exists a constant $C$ such that

$$
\|u\|_{L^{\infty}(0, H)}^{2} \leq C\left(1+\log \frac{H}{h}\right)\|u\|_{H^{1 / 2}(0, H)}^{2}, \quad \forall u \in V_{h}(0, H) .
$$

We will also need the following bound for the $H^{1 / 2}$ norm of the extension by zero from an interval to the whole $\mathbb{R}$, proved by Bramble, Pasciak, and Schatz [3, Lemma 3.5].

Lemma 4.3. There exists a constant $C$ such that for all $u \in V_{h}(0, H)$ satisfying $u(0)=u(H)=0, u=0$ outside $(0, H)$,

$$
|u|_{1 / 2, \mathbb{R}}^{2} \leq C\left(1+\log \frac{H}{h}\right)\|u\|_{L^{\infty}(0, H)}^{2}+|u|_{1 / 2,(0, H)}^{2} .
$$

An estimate of the $H^{1 / 2}$ norm of a "spike" function, obtained by sampling the value of a given function at one point, follows easily.

Lemma 4.4. There exists a constant $C$ such that for all $u \in V_{h}(0, H), 0 \leq h \leq 1$, and $v_{0} \in V_{h}(\mathbb{R})$ defined by $v_{0}(0)=u(0), v_{0}(x)=0$ for $|x| \geq h$,

$$
\left|v_{0}\right|_{1 / 2, \mathbb{R}}^{2} \leq C\left(1+\log \frac{H}{h}\right)\|u\|_{1 / 2,(0, H)}^{2} .
$$

Proof. Let $L=\|u\|_{L^{\infty}(0, H)}$. It follows from Lemma 4.3 that

$$
\left|v_{0}\right|_{1 / 2, \mathbb{R}}^{2} \leq C\left(1+\log \frac{2 h}{h}\right)\left\|v_{0}\right\|_{L^{\infty}(-h, h)}^{2}+\left|v_{0}\right|_{1 / 2,(-h, h)}^{2} .
$$


Using linearity of $v_{0}$, we obtain

$$
\left|v_{0}\right|_{1 / 2,(-h, h)}^{2}=\int_{-h}^{h} \int_{-h}^{h} \frac{\left|v_{0}(s)-v_{0}(t)\right|^{2}}{|s-t|^{2}} d s d t \leq 4 L^{2},
$$

because $\left\|v_{0}\right\|_{L^{\infty}(-h, h)}^{2}=\left|v_{0}(0)\right|^{2} \leq L^{2}$. Thus, $\left|v_{0}\right|_{1 / 2,(-h, h)}^{2} \leq C L^{2}$. But $L^{2} \leq$ $C\left(1+\log \frac{H}{h}\right)\|u\|_{1 / 2,(0, H)}^{2}$ by Lemma 4.2 , which concludes the proof.

By subtracting such spikes at the endpoints, we can extend Lemma 4.3 to the case when the values of $u$ at the endpoints are nonzero.

Lemma 4.5. There exists a constant $C$ so that for $u \in V_{h}(0, H)$ and $w \in V_{h}(\mathbb{R})$ such that $w=u$ on $[h, H-h]$, and $w(x)=0$ for $x \leq 0, x \geq H$,

$$
|w|_{1 / 2, \mathbb{R}}^{2} \leq C\left(1+\log \frac{H}{h}\right)^{2}\|u\|_{1 / 2,(0, H)}^{2} .
$$

Proof. Define $u(x)$ to be zero for $x \in(-\infty,-h) \cup(H+h, \infty)$, and linear in $[-h, 0]$ and $[H, H+h]$. Further, define $v_{0}$ and $v_{H}$ by

$$
v_{0}(x)= \begin{cases}u(0), & x=0 \\ 0, & |x| \geq h\end{cases}
$$

$v_{0}$ linear in $[-h, 0]$ and in $[0, h]$,

$$
v_{H}(x)= \begin{cases}u(H), & x=H, \\ 0, & |x-H| \geq h,\end{cases}
$$

$v_{H}$ linear in $[H-h, H]$ and in $[H, H+h]$. Writing $w$ as $w=u-v_{0}-v_{H}$, and applying Lemma 4.3 and Lemma 4.4 , we obtain

$$
\begin{aligned}
|w|_{1 / 2, \mathbb{R}}^{2} \leq C\left(1+\log \frac{H}{h}\right)\|w\|_{L^{\infty}(0, H)}^{2}+|w|_{1 / 2,(0, H)}^{2} \\
\quad=C\left(1+\log \frac{H}{h}\right)\|u\|_{L^{\infty}(0, H)}^{2}+|w|_{1 / 2,(0, H)}^{2} \\
\quad \leq C\left(1+\log \frac{H}{h}\right)\|u\|_{L^{\infty}(0, H)}^{2}+3\left(|u|_{1 / 2,(0, H)}^{2}+\left|v_{0}\right|_{1 / 2, \mathbb{R}}^{2}+\left|v_{H}\right|_{1 / 2, \mathbb{R}}^{2}\right) \\
\quad \leq C\left(\left(1+\log \frac{H}{h}\right)\|u\|_{L^{\infty}(0, H)}^{2}+|u|_{1 / 2,(0, H)}^{2}+\left(1+\log \frac{H}{h}\right)\|u\|_{1 / 2,(0, H)}^{2}\right) .
\end{aligned}
$$

Application of Lemma 4.2 to $\|u\|_{L^{\infty},(0, H)}$ concludes the proof.

We are now ready for the estimate of the $H^{1 / 2}$ norm of the glob projections $E_{G}$, which shows that an arbitrary function in $V_{h}\left(\partial \Omega_{i}\right)$ can be decomposed into its glob parts with only a small increase in the $H^{1 / 2}$ norm.

Theorem 4.6. Let $\Omega \subset \mathbb{R}^{d}, d=2$ or $d=3$. Then there exists a constant $C$ not dependent of $h$ or $H$, so that for any glob $G \in \mathcal{G}$ and for all $u \in V_{h}\left(\partial \Omega_{i}\right)$,

$$
\left\|E_{G} u\right\|_{1 / 2, \partial \Omega_{i}}^{2} \leq C\left(1+\log \frac{H}{h}\right)^{2}\|u\|_{1 / 2, G}^{2} .
$$

Proof. In the 2D case, the proposition follows by using a mapping of $\partial \Omega_{i}$ onto an interval so that $G$ maps to $(0, H)$, from Lemma 4.5 for $G$ being an edge, and from Lemma 4.4 for $G$ being a vertex. 
In the 3D case, the proposition was proved for the case of $G$ being a face of $\partial \Omega_{i}$ as Lemma 4.3 in [5]. In the case of $G$ being an edge or a vertex of $\partial \Omega_{i}$, the proof follows from Lemma 4.2. and the proof of Lemma 4.1 of [5].

The bound on the condition number of the BDD algorithm follows.

Theorem 4.7. Let $\Omega \subset \mathbb{R}^{d}, d=2$ or $d=3$, and the weight matrices $D_{i}$ be diagonal with the entries given by (16). Then there exists a constant $C$ independent of $H, h$ and $\alpha_{i}$, so that the condition number of the BDD method satisfies

$$
\text { cond }(M, S) \leq C\left(1+\log ^{2} \frac{H}{h}\right) \text {. }
$$

Proof. We only need to verify the assumption (17) of Theorem 3.3. Lemma 4.1 allows to replace the $S_{i}$ norms by the $H^{1 / 2}\left(\partial \Omega_{i}\right)$ seminorms, which may in turn be replaced by the $H^{1 / 2}\left(\partial \Omega_{i}\right)$ norms, owing to the Poincaré inequality (24). It remains to use Theorem 4.6.

\section{Computational Results}

The purpose of our computational tests was to demonstrate the fast convergence of the BDD method on complicated problems with varying coefficients.

In all of the following examples, the space $V_{h}$ of the piecewise linear functions defined on a uniform rectangular mesh of stepsize $h$ in $2 \mathrm{D}$ or 3D was used for the solution of the elliptic problem of the form (20),

$$
-\operatorname{div}(\sigma \nabla u)=1 \text { in } \Omega, \quad u=1 \text { on } \Gamma_{1}, \quad \frac{\partial u}{\partial n}=0 \text { on } \Gamma_{2},
$$

with $\partial \Omega=\Gamma_{1} \cup \Gamma_{2}, \Gamma_{1} \cap \Gamma_{2}=\varnothing$. The coefficient $\sigma$ is an elementwise constant function, and $k$ is the number of subdomains.

We have compared three algorithms: conjugate gradients applied to the reduced system (2) without preconditioning (denoted as CG in the tables), conjugate gradients with Neumann-Neumann preconditioner and the local singular problems (6) solved using the Moore-Penrose pseudoinverse (Algorithm 2.1, denoted as N-N), and conjugate gradients with the BDD preconditioner using Range $Z_{i}=\operatorname{Ker} S_{i}$ (Algorithm 2.2, denoted as BDD).

The stopping criterion for the iterations was based on

$$
\frac{\lambda_{\max }\left(M^{-1} S\right)}{\lambda_{\min }\left(M^{-1} S\right)} \frac{\left\langle M^{-1} r, r\right\rangle}{\left\langle M^{-1} b, b\right\rangle} \leq \epsilon^{2},
$$

with $r$ the current residual and $b$ the right-hand side, which guarantees the relative precision of $\epsilon$ in the energy norm, cf. Ashby, Manteuffel, and Saylor [1]. The condition number $\lambda_{\max }\left(M^{-1} S\right) / \lambda_{\min }\left(M^{-1} S\right)$ reported in the tables and also used in (27) was estimated as the ratio of the extreme Ritz values for the Krylov space, computed from the eigenvalues of a tridiagonal matrix constructed from the Lanczos recursion in conjugate gradients. Number of iterations with * means that the criterion (27) was not satisfied when the maximum number of iterations was reached.

The $2 \mathrm{D}$ examples were computed by a prototype implementation of the $\mathrm{BDD}$ method programmed using the CLAM package [24]. In the two-dimensional test BDD implementation, the weights $D_{i}$ were based on the diagonal entries of the Schur complements, as suggested in [11], because we had the diagonal entries of the 
TABLE 1. 2D results for Poisson equation on unit square (Fig. 1, $\left.\sigma_{1}=\sigma_{2}=1\right)$

\begin{tabular}{|c|c|c|c|c|c|c|c|}
\hline & & \multicolumn{2}{|c|}{ CG } & \multicolumn{2}{c|}{ N-N } & \multicolumn{2}{c|}{ BDD } \\
\cline { 3 - 8 }$h$ & iterations & cond. & iterations & cond. & iterations & cond. \\
\hline \hline $1 / 20$ & 4 & 31 & 63.426 & 10 & 45.592 & 6 & 1.231 \\
\hline $1 / 40$ & 16 & 65 & 338.008 & 38 & $3,190.710$ & 13 & 2.004 \\
\hline $1 / 50$ & 25 & 82 & 555.515 & 58 & $8,691.200$ & 13 & 2.046 \\
\hline
\end{tabular}

TABLE 2. 2D checkerboard pattern (Fig. $1, \sigma_{1}=10^{3}, \sigma_{2}=10^{-3}$ )

\begin{tabular}{|c|c|c|c|c|c|c|c|c|c|}
\hline & & & & \multicolumn{2}{|c|}{ CG } & \multicolumn{2}{|c|}{ N-N } & \multicolumn{2}{c|}{ BDD } \\
\cline { 5 - 10 }$h$ & $k$ & $\sigma_{1}$ & $\sigma_{2}$ & iter & cond. & iter & cond. & iter & cond. \\
\hline \hline $1 / 30$ & 9 & $10^{3}$ & $10^{-3}$ & 61 & 866.051 & 10 & 16.145 & 4 & 1.555 \\
\hline $1 / 40$ & 16 & $10^{3}$ & $10^{-3}$ & $130^{*}$ & $6.8910^{7}$ & 49 & $5.6110^{6}$ & 11 & 1.941 \\
\hline $1 / 50$ & 25 & $10^{3}$ & $10^{-3}$ & 116 & $1,571.66$ & 25 & 63.939 & 7 & 1.629 \\
\hline
\end{tabular}

TABLE $3.2 \times 2$ checkerboard pattern for various $\sigma_{1}, \sigma_{2}$

\begin{tabular}{|c|c|c|c|c|c|c|r|r|r|}
\hline & & & & \multicolumn{2}{|c|}{ CG } & \multicolumn{2}{|c|}{ N-N } & \multicolumn{2}{|c|}{ BDD } \\
\cline { 5 - 10 }$h$ & $k$ & $\sigma_{1}$ & $\sigma_{2}$ & iter & cond. & iter & cond. & iter & cond. \\
\hline \hline $1 / 40$ & 4 & $10^{1}$ & $10^{-1}$ & $30^{*}$ & 220 & 10 & 268 & 7 & 1.22 \\
\hline $1 / 40$ & 4 & $10^{2}$ & $10^{-2}$ & $30^{*}$ & 436 & 9 & 2,574 & 5 & 1.04 \\
\hline $1 / 20$ & 4 & $10^{4}$ & $10^{-4}$ & $30^{*}$ & 506 & 7 & 10,280 & 4 & 1.00045 \\
\hline
\end{tabular}

Schur complement available: Denote $s_{l l}^{i}$ the diagonal entry of Schur complement $S_{i}$ corresponding to global degree of freedom $l$. For subdomain $\Omega_{i}$, the weight matrix $D_{i}$ was constructed as diagonal with diagonal elements $d_{\psi_{i}(l)}^{i}$, where $\psi_{i}(l)$ is the local number in $\partial \Omega_{i}$ associated with the global degree of freedom $l$,

$$
d_{\psi_{i}(l)}^{i}=\frac{s_{l l}^{i}}{\sum_{j: l \in \partial \Omega_{j}} s_{l l}^{j}},
$$

which is essentially (16) computed node by node with the diagonal entries of $S_{i}$ used instead of the scalars $\alpha_{i}$. This choice of the weights was found to give good results [11]. The domain $\Omega$ was chosen to be the unit square and $\Gamma_{1}$ was the left-hand side of $\Omega$.

The tests show that unlike for the CG and N-N method, the condition number and the number of iterations of the BDD method does not deteriorate for increasing number of subdomains (Table 1, Fig. 1), the coefficient $\sigma$ varying by orders of magnitude between the subdomains (Table 2, Fig. 1), and increasing jumps in the coefficients (Table 3 ). 
$\Gamma_{2}$

\begin{tabular}{|c|c|c|c|c|}
\hline$\sigma_{1}$ & $\sigma_{2}$ & $\sigma_{1}$ & $\sigma_{2}$ & $\sigma_{1}$ \\
\hline$\sigma_{2}$ & $\sigma_{1}$ & $\sigma_{2}$ & $\sigma_{1}$ & $\sigma_{2}$ \\
\hline$\Gamma_{1}$ & $\sigma_{2}$ & $\sigma_{1}$ & $\sigma_{2}$ & $\sigma_{1}$ \\
\hline$\sigma_{2}$ & $\sigma_{1}$ & $\sigma_{2}$ & $\sigma_{1}$ & $\sigma_{2}$ \\
\hline$\sigma_{1}$ & $\sigma_{2}$ & $\sigma_{1}$ & $\sigma_{2}$ & $\sigma_{1}$ \\
\hline
\end{tabular}

$\Gamma_{2}$

FiguRE 1. 2D checkerboard pattern

TABLE 4. 3D Poisson's equation, various $h$ and number of subdomains

\begin{tabular}{|c|c|c|c|c|c|c|}
\hline$h_{x}$ & $h_{y}$ & $h_{z}$ & $k$ & dof & iter & cond. \\
\hline \hline $1 / 15$ & $1 / 15$ & $1 / 20$ & 36 & 5376 & 25 & 3.5375 \\
\hline $1 / 20$ & $1 / 25$ & $1 / 30$ & 120 & 9246 & 37 & 4.6354 \\
\hline $1 / 30$ & $1 / 30$ & $1 / 30$ & 27 & 29791 & 22 & 4.8000 \\
\hline
\end{tabular}

A FORTRAN implementation was used for the 3D experiments, with the action of $S_{i}$ implemented in a straightforward way following the definition of the Schur complement (3). The implementation of the action of the inverse, that is, the solution of $S_{i} y=x$, relies on the obvious fact that $y$ may equivalently be computed, using notation of $\S 2$, as solution of the sparse system

$$
\left(\begin{array}{cc}
\bar{A}_{i} & B_{i} \\
B_{i}^{T} & \dot{A}_{i}
\end{array}\right)\left(\begin{array}{c}
y \\
z
\end{array}\right)=\left(\begin{array}{c}
x \\
0
\end{array}\right),
$$


TABLE 5. 3D checkerboard pattern with alternating $\sigma$

\begin{tabular}{|c|c|c|c|c|c|c|}
\hline$h$ & $k$ & $\sigma_{1}$ & $\sigma_{2}$ & dof & iter & cond. \\
\hline \hline $1 / 25$ & 125 & $10^{0}$ & $10^{0}$ & 17576 & 22 & 3.1154 \\
\hline $1 / 25$ & 125 & $10^{1}$ & $10^{-1}$ & 17576 & 19 & 2.4893 \\
\hline $1 / 25$ & 125 & $10^{2}$ & $10^{-2}$ & 17576 & 18 & 2.2071 \\
\hline $1 / 25$ & 125 & $10^{3}$ & $10^{-3}$ & 17576 & 16 & 2.0211 \\
\hline $1 / 25$ & 125 & $10^{4}$ & $10^{-4}$ & 17576 & 16 & 2.0023 \\
\hline $1 / 25$ & 125 & $10^{5}$ & $10^{-5}$ & 17576 & 16 & 2.0002 \\
\hline $1 / 25$ & 125 & $10^{6}$ & $10^{-6}$ & 17576 & 15 & 2.0000 \\
\hline $1 / 25$ & 125 & $10^{7}$ & $10^{-7}$ & 17576 & 15 & 2.0000 \\
\hline
\end{tabular}

discarding $z$ afterwards. Since the diagonal entries of $S_{i}$ are not available, the weights were defined from $\alpha_{i}=\sigma_{i}$ by (16) with $t=1$. The problem was set on unit cube $\Omega$, with zero Dirichlet boundary condition on the whole $\partial \Omega$, and $\epsilon=10^{-18}$ was used for the stopping criterion (27). Again, the results confirm the theory.

Finally, one should note that the $l_{2}$ norm of residual of the global solution was never larger than 20 times the $l_{2}$ residual of the reduced solution. For further numerical results, see [21].

FORTRAN 77 code that implements the method is available from MGNET by anonymous ftp to casper.cs.yale.edu in the directory /mgnet/jmandel. The code invokes user-supplied subroutines that implement the matrix-vector multiplications $S_{i} x_{i}$ and solution of the possibly singular systems $S_{i} z_{i}=r_{i}$.

\section{ACKNOWLEDGEMENTS}

The authors are grateful to Olof Widlund for inspiring discussions and for providing in his papers the available theoretical tools in a systematic form that made the simple proofs presented here possible, and in particular for making available to us early drafts of [15] as well as other publications. We also thank Lawrence Cowsar for useful discussions and pointing out several improvements in an earlier version of the paper. This research was supported by NSF grants DMS-9015259, ASC-9121431, and ASC-9217394.

\section{REFERENCES}

1. S. Ashby, T. A. Manteuffel, and P. E. Saylor, A taxonomy for conjugate gradient methods, SIAM J. Numer. Anal., 27 (1990), pp. 1542-1568. MR 91i:65062

2. J.-F. Bourgat, R. Glowinski, P. Le Tallec, and M. Vidrascu, Variational formulation and algorithm for trace operator in domain decomposition calculations, in Domain Decomposition Methods, T. Chan, R. Glowinski, J. Périaux, and O. Widlund, eds., SIAM, Philadelphia, PA, 1989. MR 90b:65198

3. J. H. Bramble, J. E. Pasciak, and A. H. Schatz, The construction of preconditioners for elliptic problems by substructuring, I, Math. Comp., 47 (1986), pp. 103-134. MR 87m:65174

4. - An iterative method for elliptic problems on regions partitioned into substructures, Math. Comp., 46 (1986), pp. 361-369. MR 88a:65123

5 . - The construction of preconditioners for elliptic problems by substructuring, IV, Math. Comp., 53 (1989), pp. 1-24. MR 89m:65098

6. T. F. Chan, Analysis of preconditioners for domain decomposition, SIAM J. Numer. Anal., 24 (1987), pp. 382-390. MR 88e:65033 
7. T. F. Chan and T. P. Mathew, The interface probing technique in domain decomposition, SIAM J. on Matrix Analysis and Applications, 13 (1992), pp. 212-238. MR 92i:65183

8. P. G. Ciarlet, The Finite Element Method for Elliptic Problems, North-Holland, Amsterdam, 1978. MR 58:25001

9. L. Cowsar, J. Mandel, and M. F. Wheeler, Balancing domain decomposition for mixed finite elements. Math. Comp., 64 (1995), pp. 989-1015. MR 95j:65161

10. Y.-H. De Roeck, Résolution sur Ordinateurs Multi-Processeurs de Problème d'Elasticité par Décomposition de Domaines, PhD thesis, Université Paris IX Daupine, 1991.

11. Y.-H. De Roeck and P. Le Tallec, Analysis and test of a local domain decomposition preconditioner, in Fourth International Symposium on Domain Decomposition Methods for Partial Differential Equations, R. Glowinski, Y. Kuznetsov, G. Meurant, J. Périaux, and O. Widlund, eds., SIAM, Philadelphia, PA, 1991. CMP 91:12

12. M. Dryja, A method of domain decomposition for 3-D finite element elliptic problems, in First International Symposium on Domain Decomposition Methods for Partial Differential Equations, R. Glowinski, G. H. Golub, G. A. Meurant, and J. Périaux, eds., SIAM, Philadelphia, PA, 1988. MR 90b:65200

13. M. Dryja and O. B. Widlund, Towards a unified theory of domain decomposition algorithms for elliptic problems, in Third International Symposium on Domain Decomposition Methods for Partial Differential Equations, held in Houston, Texas, March 20-22, 1989, T. Chan, R. Glowinski, J. Périaux, and O. Widlund, eds., SIAM, Philadelphia, PA, 1990. MR 91m:65294

14. — Additive Schwarz methods for elliptic finite element problems in three dimensions, in Fifth International Symposium on Domain Decomposition Methods for Partial Differential Equations, T. F. Chan, D. E. Keyes, G. A. Meurant, J. S. Scroggs, and R. G. Voigt, eds., SIAM, Philadelphia, PA, 1992. MR 93j:65201

15. - Domain decomposition algorithms with small overlap, SIAM J. Sci.Comput., 15 (1994), pp. 604-620. MR 95d:65102

16. - Schwarz methods of Neumann-Neumann type for three-dimensional elliptic finite element problems, Comm. Pure Appl. Math, 48 (1995), pp. 121-155. CMP 95:09

17. R. Glowinski and M. F. Wheeler, Domain decomposition and mixed finite element methods for elliptic problems, in First International Symposium on Domain Decomposition Methods for Partial Differential Equations, R. Glowinski, G. H. Golub, G. A. Meurant, and J. Périaux, eds., SIAM, Philadelphia, PA, 1988. MR 90a:65237

18. D. E. Keyes and W. D. Gropp, A comparison of domain decomposition techniques for elliptic partial differential equations and their parallel implementation, SIAM J. Sci. Stat. Comput., 8 (1987), pp. s166-s202. MR 88g:65101

19. P. Le Tallec, J. Mandel, and M. Vidrascu, Balancing domain decomposition for plates, in Domain Decomposition Methods in Scientific and Engineering Computing, D. E. Keyes and J. Xu, eds., American Mathematical Society, Providence, RI, 1994, pp. 515-524. Proceedings of the 7th International Symposium on Domain Decomposition Methods, Penn State, November 1993. MR 95j: 73080

20. J. Mandel, Balancing domain decomposition, Comm. in Numerical Methods in Engrg., 9 (1993), pp. 233-241. MR 94b:65158

21. J. Mandel and M. Brezina, Balancing domain decomposition: Theory and computations in two and three dimensions, UCD/CCM Report 2, Center for Computational Mathematics, University of Colorado at Denver, November 1993.

22. J. Mandel, S. McCormick, and R. Bank, Variational multigrid theory, in Multigrid Methods, S. F. McCormick, ed., SIAM, Philadephia, 1987, ch. 5, pp. 131-177. CMP 21:05

23. M. Sarkis, Two-level Schwarz methods for nonconforming finite elements and discontinuous coefficients, in Proceedings of the Sixth Copper Mountain Conference on Multigrid Methods, Volume 2, N. D. Melson, T. A. Manteuffel, and S. F. McCormick, eds., no. 3224, Hampton VA, 1993, NASA, pp. 543-566.

24. Scientific Computing Associates, CLAM User's Guide; The Computational Linear Algebra Machine, Version 2.00, New Haven, CT, 1990.

25. B. F. Smith, A domain decomposition algorithm for elliptic problems in three dimensions, Numer. Math., 60 (1991), pp. 219-234. MR 92m:65159 
26. O. B. Widlund, An extension theorem for finite element spaces with three applications, in Numerical Techniques in Continuum Mechanics, W. Hackbusch and K. Witsch, eds., Braunschweig/Wiesbaden, 1987, Notes on Numerical Fluid Mechanics, v. 16, Friedr. Vieweg und Sohn, pp. 110-122. Proceedings of the Second GAMM-Seminar, Kiel, January, 1986.

27. — Iterative substructuring methods: Algorithms and theory for elliptic problems in the plane, in First International Symposium on Domain Decomposition Methods for Partial Differential Equations, R. Glowinski, G. H. Golub, G. A. Meurant, and J. Périaux, eds., SIAM, Philadelphia, PA, 1988. MR 90c:65138

Center for Computational Mathematics, University of Colorado at Denver, Denver, COLORAdO 80217-3364

E-mail address: jmandel@colorado.edu

E-mail address: mbrezina@carbon.denver.colorado.edu 